\title{
Investigating the Phytochemicals and Antimicrobial Properties of Three Sedge (Cyperaceae) Species
}

\author{
Tiwalade Adeyemi ADENIYI ${ }^{1 *}$, Peter A. ADEONIPEKUN ${ }^{1}$, Elizabeth A. OMOTAYO² \\ ${ }^{1}$ University of Lagos, Department of Botany,Akoka,Nigeria; dessytwa@yahoo.com (*corresponding author); pladeonipekun@yahoo.com \\ ${ }^{2}$ University of Lagos, Department of Microbiology, Akoka, Nigeria; elizabethomotayo@yahoo.com
}

\begin{abstract}
In order to evaluate the medicinal value of notorious sedge weeds, three species: Cyperus esculentus, Cyperus rotundus and Mariscus alternifolius were investigated for their phytochemical constituents and antimicrobial properties. Preliminary qualitative phytochemical constituents and in vitro antimicrobial activities were evaluated against four fungi species: Aspergillus niger, Aspergillus fumigatus, Penicillium chrysogenum and Candida albicans, and three bacteria species: Escherichia coli, Salmonella typhi and Staphylococcus aureus. Two solvents, water and ethanol, were used to produce the extracts and were screened for their antimicrobial activity. Antimicrobial activity evaluation of the extracts against pathogens was carried out at $100 \mathrm{mg} / \mathrm{ml}$ concentration by Disc Diffusion method for fungi, Disc Diffusion and Agar Well Diffusion methods for bacteria. Observed activities were related to standard antibiotics, antifungal and antibacterial, which served as controls. Phytochemically, the plant extracts showed the presence of carbohydrates, flavonoids, ketose sugars, steroids, reducing sugars and tannins. The ethanolic extract of $C$. rotundus exhibited the highest activity against $A$. niger, $E$. coli and $S$. aureus. No extract was active against $C$. albicans. From these findings, it was concluded that $C$. rotundus is a potential source of bioactive compounds for new drugs upon isolation and purification for treating infections caused by these pathogens.
\end{abstract}

Keywords: bacteria, Cyperus esculentus, Cyperus rotundus, plant extract, fungi, Mariscus alternifolius

\section{Introduction}

Nearly $80 \%$ of the world population depends upon traditional system of health care (Ganesan et al., 2004). The indigenous traditional knowledge of medicinal plants of various ethnic communities, where it has been transmitted orally for centuries, is fast disappearing from the face of the earth due to the advent of modern technology and transformation of traditional culture (Ganesan et al., 2004). However, during the last few decades there has been an increasing interest in the study of medicinal plants and their traditional uses in different parts of the world. This led to major pioneering works such as the isolation of quinine from Cinchona spp. (bark extracts), reserpine from Rauvolfia serpentine - serpent wood (root extracts) and diosgenin from Dioscorea spp. (tuber extracts) (Joy et al., 2001). Consequently, the level of awareness of phytomedicine in Nigeria is very large. It is estimated that more than $80 \%$ of the Nigerian population use herbal remedies in one form or the other, because less than $35 \%$ of the population has access to modern health care facilities (Awosika, 1993). Thus, medicinal plants form the basis of primary health care for majority of the people living in the rural and remote areas in Nigeria (Awosika, 1993). Most recently, the efforts of some newspaper columnists in raising awareness over herbal medicine efficacy in curing diseases cannot be over-simplified. The availability of many of these herbal plants makes them more readily affordable to the rural and even urban dwellers. Of particular significance in herbal medicine are common weeds around homes and waste-lands, as well as farmlands where sedges form important components.

Among these common weeds is the family Cyperaceae which are ubiquitous, but may be difficult to recognize because of their closeness to the grass family, with the exception of their triangular shaped stems, that are characteristic. Lowe and Stanfield (1974) remarked that about four-fifths of the species grow in damp or wet places (including a few submerged aquatics), while one-fifth is found in drier regions, such as savannah grassland and sandy places including sand-dunes.

Cyperus esculentus L. (Family: Cyperaceae, common name: Tiger nut, Nigerian local names: Yoruba - Ofio, Hausa - Ayaya and Igbo - Akiausa). It is found in all tropical and warm-temperate regions except Malaysia, Australia and Oceania. In Nigeria, it is commonly found across the country. It is a weed found in cultivated areas, in moist places and waste-lands (Burkill, 1985). It is $24-55 \mathrm{~cm}$ tall, producing rhizomes from the base which are $2 \mathrm{~mm}$ thick 
and bear tubers. Stem is triangular, with sides approximately $2 \mathrm{~mm}$ wide. Leaves are fairly numerous at the base of the stem, reaching $30 \mathrm{~cm}$ long and $8 \mathrm{~mm}$ wide, but often narrower. Inflorescence subtended by approximately four bracts, the longest approximately $20 \mathrm{~cm}$ long and $5 \mathrm{~mm}$ wide, usually tapering to a fine point. Inflorescence is a simple umbel when small, or a compound umbel when larger, with primary rays of 4-9 $\mathrm{cm}$ long, secondary rays if present are $2 \mathrm{~cm}$ long. Branches ending in spikes $1-3 \mathrm{~cm}$ long, composed of 6-25 golden-yellow spikelets, each 7-15 $\mathrm{mm}$ long, 1-1.5 mm broad, with 3-8 glumes along each side. Glumes approximately $3 \mathrm{~mm}$ long, with a fairly prominent midrib, and with a papery edge. They are occasionally viviparous (Lowe and Stanfield, 1974). Medicinally, they are used in healing menstrual discomforts and stomach troubles (Burkill, 1985).

Cyperus rotundus L. (Family: Cyperaceae, common name: Nut grass, Nigerian local names: Hausa - Giragiri, Fulfude - Ayaare and Yoruba - Danda). It is found in pantropical and sub-tropical regions in West Africa, and other damp places. Its distribution ranges from Mali to Niger, and towards Nigeria, Ghana and Sierra Leone. It is also found in warm temperate regions often as a rice-field weed (Burkill, 1985). In Nigeria it is found across the country (Lowe and Stanfield, 1974). Its rhizome is fairly narrow with fibrous brown scales. Stems are $20-40 \mathrm{~cm}$ high, $1-2 \mathrm{~mm}$ thick, with a swollen tuberous base. Leaves vary in length from half as long as the stem to equal the length of the stem in some cases; leaves are sometimes few (6) and narrow $(2 \mathrm{~mm})$; sometimes numerous (14) and broader $(5 \mathrm{~mm})$. Inflorescence is $3-8 \mathrm{~cm}$ long, often rather narrow with suberect spikelets, subtended by approximately 3 bracts similar to the leaves, and from $1 / 2$ to $1 \frac{1}{2}$ times as long as the inflorescence. Inflorescence usually with a few primary rays $2-5 \mathrm{~cm}$ long, ending in a short spike of 3-10 spikelets, sometimes with 1 or 2 short secondary rays at the base of the spike. Spikelets are rather long and flat, generally reddishbrown, $12-20 \mathrm{~cm}$ long, $1.5-2 \mathrm{~mm}$ broad, with 5-10 glumes along each side. Glumes with a green keel and red-brown sides, 3-4 mm long (Lowe and Stanfield, 1974). In medicine, tubers have been reported to be used in treating diarrhea and dysentery; as genital stimulants or depressants, treatment of kidney troubles, stomach troubles and as diuretics (Burkill, 1985). The tubers also possess aromatic substances that are used as insecticides and arachnicides (Burkill, 1985). The presence of polyphenol, flavonol glycoside, saponin, vitamin $\mathrm{C}$, sesquiterpenoids and essential oil from phytochemical investigation of $C$. rotundus rhizomes was reported by Nagulendran $e t$ al. (2007).

Mariscus alternifolius Vahl. (Family: Cyperaceae, common name: Mariscus, Nigerian local names: Hausa Ayaa, Yoruba - Alubosa eranko and Igbo - Ataku mainya). It is pan-tropical and common in damp grassy places in Togo, Gambia, Nigeria, Liberia, Ivory Coast, Ghana, Gambia, Guinea and Cameroon (Burkill, 1985). It is found across Nigeria (Lowe and Stanfield, 1974). Mariscus is perhaps the most common species of sedge in Nigeria, except the far north. An extremely variable plant, forming shoots often thickened or almost bulb-like at the base, sometimes from a short woody rhizome, with numerous fibrous roots. May be very slender, or may reach $1 \mathrm{~m}$ tall with leaves $8 \mathrm{~mm}$ wide.
Leaf sheaths are purplish-red. Stems rounded to triangular. Inflorescence is simple umbel with a different number of rays, the longest approximately $10 \mathrm{~cm}$ long, and subtended by leafy bracts. Spikelets always densely crowded into heads, which may be almost globose and approximately $5 \mathrm{~mm}$ diameter, or elongated into spikes $3 \mathrm{~cm}$ long and $1 \mathrm{~cm}$ wide. In young inflorescences, the heads are almost sessile, but stalks (i. e. the rays of the inflorescence) soon develop to various lengths. Spikelets usually under $5 \mathrm{~mm}$ long with 1 or 2 ribbed glumes showing; in some specimens there may be spikelets over $5 \mathrm{~mm}$ long, with 2 or 3 glumes visible (Lowe and Stanfield, 1974). The chewed stem bandaged on a cut or wound for at least three days is reported to have a healing effect. The swollen stem base after washing, ground in a mortar and then mixed into some honey is a treatment for gonorrhea (Burkill, 1985).

To verify the claimed and reported medicinal uses of some sedges, the antimicrobial properties and phytochemicals present in the extracts of Cyperus esculentus, Cyperus rotundus and Mariscus alternifolius were investigated.

\section{Materials and methods}

Plant collection

Plant samples were collected from the University of Lagos Campus, Akoka, Lagos State and neighbouring surroundings. The plants were collected wholly with roots, shoots and inflorescences. The plants were confirmed in the Herbarium of the Department of Botany, University of Lagos. The collected plants are Cyperus esculentus, $C$. rotundus and Mariscus alternifolius.

\section{Test microorganisms}

The test bacteria: Escherichia coli, Staphylococcus aureus and Salmonella typhi were obtained from Lagos University Teaching Hospital, Idi-Araba, Lagos and the test fungi: Aspergillus niger and Aspergillus fumigatus were obtained from Microbiology Department, University of Lagos. However, Candida albicans and Penicillium chrysogenum were obtained from the Nigerian Institute for Medical Research, Yaba, Lagos. The bacteria strains were maintained on Nutrient Agar while the fungi were maintained on Potato Dextrose Agar at $4{ }^{\circ} \mathrm{C}$ in a refrigerator.

\section{Preparation of plant extracts}

The plant materials were cut into pieces and air-dried for a week in an oven. Dried samples were ground into powder with a grinding machine. For the aqueous extraction, $50 \mathrm{~g}$ of each powdered plant sample was soaked in $500 \mathrm{ml}$ of cold distilled water in a conical flask and left undisturbed for 48 hours. Thereafter, the extracts were filtered off using Whatman No 1 filter paper. The filtrate was concentrated under vacuum below $40^{\circ} \mathrm{C}$ using a rotary evaporator (Bag et al., 2009). For organic solvent extraction, $50 \mathrm{~g}$ of air-dried powder was soaked in $500 \mathrm{ml}$ of ethanol in a conical flask and left undisturbed for 48 hours. Thereafter, the extract was filtered off using Whatman No 1 filter paper. The filtered extract was concentrated under vacuum below $40^{\circ} \mathrm{C}$ using a rotary evaporator (Bag et al., 2009). The extracts thus obtained were stored in well- labeled sterile bottles and kept in the freezer at $4{ }^{\circ} \mathrm{C}$ until further 
278

use for the screening of antimicrobial activity.

\section{Antimicrobial Assay}

Antifungal Activity Test of Plant Extracts

This was done using the Agar Diffusion Test Method of Irobi and Daramola (1993).

About $10 \mathrm{ml}$ of Potato Dextrose Agar (PDA) was poured into petri dishes and allowed to solidify. Liquid inoculum was prepared by pouring cooled sterile distilled water on already grown fungal plates. The suspension was transferred into a test tube. This allowed for fungal spores to be easily removed from the pure culture. A drop pipette was used to transfer two drops of fungal inoculum into each of the agar plates. A sterile glass rod was used for even spreading. To prepare discs, Whatman No 1 filter paper was perforated using a paper punch to form paper discs $6 \mathrm{~mm}$ in diameter. The discs were wrapped in aluminum foil and sterilized in an autoclave at $121^{\circ} \mathrm{C}$ for 15 minutes. After sterilization, the discs were dropped in each extract (100 $\mathrm{mg} / \mathrm{ml})$.

Two controls were used: sterile distilled water and Fulcin. Fulcin control was prepared by dissolving $12.5 \mathrm{~g}$ of Fulcin powder in $10 \mathrm{ml}$ of sterile distilled water. Four of the discs from each of the three extracts, antibiotic and water controls were aseptically placed with the aid of a sterile forceps on PDA and inoculums in petri dishes. For each fungus, two replicates were produced by repeating the above processes. This is to reduce experimental errors by calculating the average of the values obtained. All the plates containing the extracts and fungi were incubated at $25^{\circ} \mathrm{C}$. The zones of inhibition were measured after 48 hours of incubation and the results analyzed statistically.

\section{Antibacterial Activity Test of Plant Extracts}

\section{Disc Diffusion Assay}

This was done using the Agar Diffusion Test Method of Bauer et al. (1966). About $10 \mathrm{ml}$ of Nutrient Agar (NA) was poured into petri dishes and allowed to solidify. Petri dishes were inoculated by streaking bacteria over their surfaces with the aid of an inoculating loop. To prepare discs, Whatman No 1 filter paper was perforated using a paper punch to form paper discs of $6 \mathrm{~mm}$ in diameter. The discs were wrapped in aluminum foil and sterilized in an autoclave at $121^{\circ} \mathrm{C}$ for 15 minutes. After sterilization, they were dropped in each extract $(100 \mathrm{mg} / \mathrm{ml})$.
Two controls were used: sterilized distilled water and Tetracycline - an antibiotic. Tetracycline control was prepared by dissolving $12.5 \mathrm{~g}$ of tetracycline powder in 10 $\mathrm{ml}$ of sterile distilled water. Four of the discs from each extract, antibiotic and water control were then aseptically placed with the aid of sterile forceps on petri dishes with NA and inocula. For each bacterium, the experiment was performed in duplicates. All the plates containing the extracts and bacteria were then incubated at $37^{\circ} \mathrm{C}$. The zones of inhibition around the disc were measured after 24 hours of incubation and the results analyzed statistically.

\section{Agar Well Diffusion Assay}

The Agar Diffusion Test Method of Aneja and Joshi (2009) was applied. About $10 \mathrm{ml}$ of Nutrient Agar (NA) was poured into petri dishes and allowed to solidify. The medium was inoculated with bacteria by streak plate method. Wells were prepared in the plates with a cup-borer $(0.85 \mathrm{~cm})$ and $100 \mu \mathrm{l}$ of the test compound was pipetted directly into the well. This was repeated for all the extracts and controls. Two controls were used: sterilized distilled water and Ciprofloxacin. Ciprofloxacin control was prepared by dissolving $12.5 \mathrm{~g}$ of ciprofloxacin powder in 10 $\mathrm{ml}$ of sterile distilled water. Prior to incubation at $37^{\circ} \mathrm{C}$ for 24 hours, the petri dishes were kept at room temperature for 15 minutes in order to promote diffusion of the extracts into the agar. All the tests were made in duplicates and the mean diameter of the inhibition zones were analyzed statistically.

\section{Phytochemical Screening}

Phytochemical screenings of the plant samples were carried out qualitatively using the method described by Harbone (1998). These screenings are: Fehling's Solution test for reducing sugars, Molisch test for carbohydrate sugars, Barfoed's test for monosaccharides, Resorcinol test (or Seliwanoffs test) for keto-sugars, Phloroglucinol test for pentose sugars, test for Alkaloids, general test for Glycosides, tests for saponin, tannins, anthraquinones, flavonoids, steroids and terpenoids.

\section{Statistical Analysis}

Tests were carried out in duplicate for the three separate experiments. Analysis of the standard error was used for comparison between the mean values of the studies parameters.

Tab. 1. Effects of water and ethanolic extracts of sedges on fungi

\begin{tabular}{|c|c|c|c|c|}
\hline \multirow{2}{*}{ Extracts } & \multicolumn{4}{|c|}{ Inhibition zones of fungi $(\mathrm{cm})$} \\
\hline & A. niger & A. fumigatus & P. chrysogenum & C. albicans \\
\hline C. esculentus (water extract) & na & $1.00 \pm 0.38$ & $4.00 \pm 0.85$ & na \\
\hline C. esculentus (ethanol extract) & na & $4.63 \pm 1.18$ & $6.13 \pm 1.22$ & na \\
\hline C. rotundus (water extract) & na & $7.88 \pm 1.73$ & $0.88 \pm 0.74$ & na \\
\hline C. rotundus (ethanol extract) & $9.38 \pm 0.98$ & na & $2.38 \pm 1.08$ & na \\
\hline M. alternifolius (water extract) & na & $4.88 \pm 1.39$ & $2.38 \pm 0.71$ & na \\
\hline M. alternifolius (ethanol extract) & na & na & $6.75 \pm 0.94$ & na \\
\hline Fulcin & $10.00 \pm 0.33$ & $13.88 \pm 0.72$ & $11.63 \pm 0.38$ & $20.00 \pm 0.42$ \\
\hline Water & na & na & na & na \\
\hline
\end{tabular}

${ }^{*}$ na $=$ not active. Data are presented as the mean \pm SE of each duplicate test. 
Tab. 2. Effects of water and ethanolic extracts of sedges on bacteria (disc method)

\begin{tabular}{|c|c|c|c|}
\hline \multirow{2}{*}{ Extracts } & \multicolumn{3}{|c|}{ Inhibition zones of bacteria $(\mathrm{cm})$} \\
\hline & Escherichia coli & Staphylococcus aureus & Salmonella typhi \\
\hline C. esculentus (water extract) & $2.13 \pm 0.79$ & $0.75 \pm 0.41$ & $0.75 \pm 0.49$ \\
\hline C. esculentus (ethanol extract) & $1.38 \pm 0.80$ & $1.25 \pm 0.49$ & $0.25 \pm 0.16$ \\
\hline C. rotundus (water extract) & $2.00 \pm 0.68$ & $0.50 \pm 0.19$ & $0.50 \pm 0.50$ \\
\hline C. rotundus (ethanol extract) & $6.75 \pm 0.77$ & $2.25 \pm 0.56$ & na \\
\hline M. alternifolius (water extract) & $0.63 \pm 0.38$ & $0.50 \pm 0.38$ & $1.00 \pm 0.57$ \\
\hline M. alternifolius (ethanol extract) & $0.50 \pm 0.33$ & $1.00 \pm 0.50$ & $0.13 \pm 0.13$ \\
\hline Tetracycline & $19.75 \pm 0.98$ & $31.00 \pm 1.13$ & $20.00 \pm 0.87$ \\
\hline Water & na & na & na \\
\hline
\end{tabular}

${ }^{*}$ na $=$ not active. Data are presented as the mean \pm SE of each triplicate test.

\section{Results}

\section{Antimicrobial results}

\section{Phytochemical results}

Cyperus esculentus contained the highest number of phytochemicals; $C$. rotundus contained tannins and reducing sugars, while $M$. alternifolius contained relatively the least number of phytochemicals (only reducing sugars). Alkaloids, saponins, anthraquinones and terpenoids were not found in the three sedges (Tab. 3).

Tab. 3. Phytochemical screening of sedges extracts

\begin{tabular}{cccc}
\hline \multirow{2}{*}{ Test } & \multicolumn{3}{c}{ Sedges } \\
\cline { 2 - 4 } & C. esculentus & C. rotundus & M.alternifolius \\
\hline Fehling's & - & + & + \\
Molisch & + & - & - \\
Barfoed's & - & - & - \\
Resorcinol & + & - & - \\
Phloroglucinol & - & - & - \\
Alkaloids & - & - & - \\
(Dragendoffs) & - & - & - \\
Saponin & - & + & - \\
Tannins & + & - & - \\
Anthraquinones & - & - & - \\
Flavonoids & + & - & - \\
Steroids & + & - & - \\
Terpenoids & - & & \\
\hline
\end{tabular}

Key: Inference of the test is indicated in parenthesis: $(+)$ indicates present; $(-)$ indicates absent.

\section{Discussion}

Burkill (1985) observed that nuts of Cyperus esculentus and $C$. rotundus have medicinal properties which have been harnessed by traditional medicine practitioners, but only a few of these properties have been proven scientifically. However, in this present study, only the shoots, roots and inflorescence of Cyperus esculentus and C. rotundus were utilized. This was done to further assess other floral parts for their medicinal importance, due to their fast growth rate which make them more readily available for use, since the tubers take a longer time to develop. Traditional medicine practitioners make use of water primarily as a solvent, but studies have shown that alcohol extracts of plants are much better and powerful. This may be due to the better solubility of the active components in organic solvent (De Boer et al., 2005). The two extracts (ethanolic and aqueous) used in this study showed either inhibitory or no activities against the different species of fungi and bacteria tested. $A$. niger is one of the most common species of Aspergillus that causes aspergillosis in humans. Aspergillosis develops in the lungs of humans who are immuno-compromised and results in symptoms such as cough, fever, chest pains and breathing difficulties. In plants, Aspergillus niger causes black mould of onion bulbs. Due to the high potency of Cyperus rotundus (ethanolic extract) against $A$. niger, it can be suggested that isolation of the bioactive compounds responsible for the inhibitory activity and the purification of this compound may provide another reliable antimicrobial just like Fulcin, which will be used specifically for controlling $A$. nigerrelated infections in humans and plants.

The inactivity of $C$. rotundus (ethanolic extract) against A. fumigatus and its potency against $A$. niger may be attributed to the fact that both species produce different mycotoxins. Aspergillus fumigatus produces a mycotoxin known as gliotoxin, which is different from ochratoxin produced by $A$. niger. Aspergillus fumigatus is the most common cause of aspergillosis in the lungs of immunocompromised humans. Its effectiveness is as a result of the mycotoxin - gliotoxin, which is genotoxic and cytotoxic (Susana et al., 2002). Aqueous extract of C. rotundus was the most effective plant extract against the fungus $(7.88 \pm 1.73 \mathrm{~cm})$. This suggests that further isolation and purification of the bioactive compounds responsible for the inhibitory activity may lead to the development of effective drugs which can be used as a combination therapy with Fulcin against the fungus.

Penicillium chrysogenum is a wonderful source of antibiotic - Penicillin; it causes no diseases in humans, except few cases of allergies, but may cause mould on plant stored products. All plant extracts showed relatively mild inhibitory activities against Penicillium chrysogenum compared to the antifungal control - Fulcin (Tab. 1). This is probably due to the fact that the fungus itself produces an antibiotic contributing to its weak potency and susceptibility. The resistance of Candida albicans (Tab. 1) to most antimicrobial agents was clearly demonstrated in this study as no extract showed inhibitory activity against the yeast-like fungus. Candida albicans is the causative agent of candidiasis: an opportunistic infection very difficult to control in immuno-compromised patients, but easily cured in patients not immuno-compromised. According to Awodele et al. (2007), the use of some common disinfectants such as "savlon" and "jik" against C. albicans 
280

revealed that unless $100 \%$ concentration of these substances are used, $C$. albicans cannot be controlled.

The ethanolic extract of $C$. rotundus was the most active plant extract against E. coli $(6.75 \pm 0.77 \mathrm{~cm}$, Tab. 2$)$ and this probably supports the reported use of the plant by traditional medicine practitioners in treating diarrhoea and other stomach troubles. Isolation and purification of the bioactive compounds responsible for this inhibitory activity are necessary to produce more antibiotics which can be used effectively against $E$. coli. Cyperus rotundus ethanolic extract also showed the highest inhibitory action against $S$. aureus $(2.25 \pm 0.56 \mathrm{~cm})$. This further enunciates the anti-microbial potency of this plant extract. The relatively low inhibitory activities of the plant extracts against Salmonella typhi compared to the antibiotic control - Tetracycline demonstrates the difficulty involved in controlling Salmonella typhi.

Mariscus alternifolius ethanolic extract was the most active against $E$. coli, and it showed similar results to $C$. rotundus. The authors are not aware of any reported use of Mariscus alternifolius in treating stomach troubles or diarrhoea. Further purification and isolation of the active compounds in this extract are required for pharmacological evaluation. This plant may be a potential source of a new type of antibiotic. The ethanolic and aqueous extracts of $C$. rotundus showed the highest inhibitory activities against $S$. aureus compared to the other four plant extracts. This result again further illustrates the high potency of $C$. rotundus against micro-organisms. The ethanol extract of $C$. esculentus was the most active against Salmonella typhi and the result was similar to that obtained from the antibiotic control - Ciprofloxacin. This indicates the high potency of the plant extract. Isolating and purifying the bioactive compounds may lead to the development of another suitable antibiotic against $S$. typhi.

The observed mild inhibitory activities of most of the tested extracts on the growth of the microbes possibly suggest that the use of these extracts at higher concentrations may be very effective. This is indicated by their use by the traditional medicine practitioners. Amongst the gram-positive and gram-negative bacteria, the grampositive bacterium (Staphylococcus aureus) was more susceptible to the extracts with $100 \%$ susceptibility as compared to the gram-negative bacteria (Salmonella typhi and Escherichia coli) $66.7 \%$ and $91.8 \%$ susceptibility respectively. This observation agrees with previous works that plant extracts are more active against gram-positive bacteria than gram-negative bacteria (Vlietinck et al., 1995; Rabe and Van Staden, 1997).

The result of phytochemical screening showed that $C$. esculentus contained a higher number and variety of phytochemicals than $C$. rotundus and Mariscus alternifolius. $C$. esculentus had a record of five phytochemicals (carbohydrates, ketose sugars, tannins, flavonoids and steroids), C. rotundus and Mariscus alternifolius had two (tannins and reducing sugars) and one (reducing sugars) respectively (Tab. 3). According to the antimicrobial assays, it can be suggested that most of the phytochemicals in $C$. esculentus do not possess bioactive forms except against $S$. typhi. The steroids, carbohydrates and ketose sugars may be responsible for this activity because these compounds are used in the production of phytoalexins in plants. Phytoalexins are antimicrobial substances synthesized by plants that accumulate rapidly at areas of incompatible pathogen infection (Yukihiro et al., 2002). The presence of tannins and flavonoids suggests that the class of tannins present in this plant is made up of non-hydrolysable or condensed tannins, which are effective anti-infective compounds (Okigbo et. al., 2009). Further research needs to be conducted in this area to determine the phytoalexins and tannins present in this plant through their isolation and purification. C. rotundus contained reducing sugars and tannins. The class of tannins may be responsible for the anti-diarrhoeal potential of the plant (Akiyama et al., 2001).

Also, the combination of the reducing sugars constitute a building block for the production of phytoalexins and this may be responsible for the high antimicrobial potency of the plant (Yukihiro et al., 2002). Mariscus alternifolius contained only reducing sugars. This phytochemical is probably responsible for the plants' high inhibitory activity against $E$. coli. Also, further research needs to be done on this plant to exploit its antimicrobial potential. None of the plant extracts contained alkaloids, anthraquinones, saponins and terpenoids, which have been reported in other plants with anti-microbial properties (Opara et al., 2012) suggesting the diversity of possible anti-microbial drugs.

\section{Conclusions}

Cyperus esculentus, Cyperus rotundus and Mariscus alternifolius could be important sources of natural antimicrobials. The results of the present study support the traditional usage of the studied plants in the treatment of diarrhoea and stomach troubles. This suggests that some of the plant extracts possess compounds with antimicrobial properties that can be used as antimicrobial agents in new drugs for the therapy of infectious diseases caused by pathogens. The most active extracts can be subjected to isolation of the therapeutic antimicrobials and further pharmacological evaluation carried out on them. Among these sedge species, particularly $C$. rotundus is potential sources of bioactive compounds needed in the synthesis of antibiotics against the tested pathogens.

\section{Acknowledgements}

We appreciate the efforts of the Department of Botany, Department of Microbiology, Department of Pharmacognosy, University of Lagos, for the provision of microorganisms and laboratory work assistance. We also appreciate the effort of Nigerian Medical Research, Yaba, Lagos.

\section{References}

Akiyama H, Fujii K, Yamasaki O, Oono T, Iwatsuki K (2001). Antibacterial activity of several tannins against Staphylococcus aureus. J Antimicrob Chemother 48 (4):487-491.

Aneja K, Joshi R (2009). Antimicrobial activity of Amomum subulatum and Elettaria cardamomum against dental caries-causing microorganisms. Ethnobot Leaflets 7:3. 
Awodele O, Emeka P, Agbamuche H, Akintonwa A (2007). The antimicrobial activities of some commonly used disinfectants on Bacillus subtilis, Pseudomonas aeruginosa and Candida albicans. Afr J Biotechnol 6(8):987-990.

Awosika F (1993). Traditional medicine as the solution to Nigeria health problems. Clin Pharm and Herbal Medicine 9(3):26-31.

Bag A, Bhattacharya S, Bharati P, Pal N, Chattopadhyay R (2009). Evaluation of antibacterial properties of chebulicmyrobalan (fruit of Terminalia chebula Retz.) extracts against methicillin resistant Staphylococcus aureus and trimethoprimsuphamethoxazole resistant uropathogenic Escherichia coli. Afr J Plant Sci 3(2):25-29.

Bauer A, Kirby W, Sherris J, Turck M (1966). Antibiotic susceptibility testing by a standardized single disk method. Am J Clin Pathol 45:493-496.

Burkill H (1985). The useful plants of West Tropical Africa: Royal Botanical Garden, Chicago.

De Boer H, Kool A, Broberg A, Mziray W, Hedberg I, Levenfors J (2005). Antifungal and antibacterial activity of some herbal remedies from Tanzania. J Ethnopharmacol 96:461-469.

Ganesan S, Suresh N, Kesaven L (2004). Ethnomedicinal survey of Lower Palani Hills of Tamilnadu. Indian J Tradit Know 3 (3):299-304.
Harbone J (1998). Phytochemical methods: A guide to modern techniques of plant analysis: Chapman and Hall, London.

Irobi O, Daramola S (1993). Antifungal activities of crude extracts of Mitracorpus villous (Rubiaceae). J Ethnopharmacol 40:137-140.

Joy P, Thomas J, Mathew S, Skaria B (2001). Tropical Horticulture. In: Medicinal Plants. Naya Prokash, Calcutta.

Lowe J, Stanfield D (1974). The Flora of Nigeria: Sedges (Family Cyperaceae): Ibadan University Press, Ibadan.

Nagulendran K, Velavan S, Mahesh R, Hazeena V (2007). In vitro antioxidant activity and total polyphenolic content of Cyperus rotundus rhizomes. Electron J Ch 4(3):440-449.

Okigbo R, Anuagasi C, Amadi J (2009.) Advances in selected medicinal and aromatic plants indigenous to Africa. J Med Plants Res 3(2):86-95.

Opara F, Anuforo H, Okechukwu R, Mgbemena I, Akujobi C, Adjero A (2012). Preliminary phytochemical screening and anti-bacterial activities of leaf extracts of Terminalia catappa. J Emerg Trends Eng Appl Sci 3(3):424-428.

Rabe T, Van Staden J (1997). Antibacterial activity of South African plants used for medicinal purposes. J Ethnopharmacol 56:81-87. 